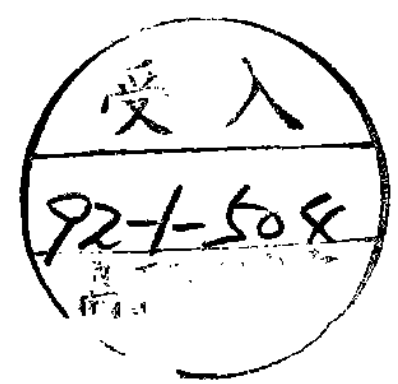

CERN-TH. 6263/91

\title{
A Kallosh Theorem for BF-Type Topological Field Theory
}

\author{
Danny Birmingham ${ }^{1}$ \\ CERN, Theory Division, CH-1211, Geneva 23, Switzerland \\ Richard Gibbs and Soussan Mokhtari \\ Physics Department, Louisiana Tech University, \\ Ruston, LA 71272, USA
}

\begin{abstract}
A Kallosh theorem is established for the case of $B F$-type theories in threedimensions, including a coupling to Chern-Simons theory. The phase contribution to the one-loop off-shell effective action is computed for a 2-parameter family of local covariant gauges. It is shown that the phase is independent of these parameters, and thus equals the "no Vilkovisky-DeWitt" gauge result. The field space metric dependence of a corresponding calculation for generalized $B F$ theory is briefly discussed.
\end{abstract}

CERN-TH. 6263/91

September 1991

\footnotetext{
${ }^{1}$ Supported by "Commission des Communautés Européennes" (DG XII-CCR)
} 


\section{Introduction}

Recently, progress has been made in understanding the structure of the one-loop offshell effective action in three-dimensional $B F$-type topological field theory $[1,2,3]$. The central object of interest in such a study is the $\eta$-function of the determinant of a first order operator. The most natural framework for examining these theories is provided by the Vilkovisky-DeWitt effective action $[4,5]$.

According to the tenets of this program, one must ensure that the geometry on the space of fields is accounted for in an appropriate manner. It was observed in [3] that the presence of the $\sqrt{\operatorname{det} G_{i j}}$ term in the functional measure had a significant effect on the result obtained for the $\eta$-function, despite its field independence. Having incorporated this factor, it was found that the $\eta$-function contribution to the effective action was proportional to the pure Chern-Simons term, in agreement with a diagrammatical analysis of [1]. The calculation in [3] was performed in the so-called "no VD" gauge; in such a gauge one can show that potential VD correction terms vanish. As such, it represents the unique (i.e. gauge fixing independent) result within this program.

In the present paper, we would like to explicitly demonstrate the gauge-fixing independence of the above results. Our aim is to establish a Kallosh-type theorem [6] for the $\eta$-function of this theory. This involves the introduction of a 2-parameter family of covariant gauges; in other words, the "no VD" gauge is implemented in the path integral with the use of two arbitrary parameters. Since we are dealing here with off-shell contributions to the effective action, the Kallosh theorem states that the $\eta$-function should be independent of these gauge fixing parameters.

The outline of this article is as follows: The theory under study, namely, $B F$ theory $[7,8,9]$ coupled to Chern-Simons theory $[10]$ is first presented, together with the relevant details of its quantization. We then recall the construction of an appropriate field space metric and summarize the calculational method being employed. The Kallosh theorem is then explicitly verified, and we conclude with some brief remarks on similar calculations in a generalized $B F$ theory [11].

\section{The Model}

The classical action of the theory we wish to study is given by:

$$
S_{c}=\int d^{3} x \operatorname{tr} \epsilon^{\alpha \beta \gamma} B_{\alpha} F_{\beta \gamma}+\frac{k}{4 \pi} \int d^{3} x \operatorname{tr} \epsilon^{\alpha \beta \gamma}\left(A_{\alpha} \partial_{\beta} A_{\gamma}+\frac{2}{3} A_{\alpha} A_{\beta} A_{\gamma}\right)
$$

which corresponds to a coupling of $B F$ theory to the Chern-Simons term. The results we establish are also valid in the limit in which the integer-quantized Chern-Simons coupling $k$ is set to zero. The field content consists of a gauge connection $A_{\alpha}$ with curvature $F_{\alpha \beta}=$ $\partial_{\alpha} A_{\beta}-\partial_{\beta} A_{\alpha}+\left[A_{\alpha}, A_{\beta}\right]$, while $B=B_{\alpha}^{a} T^{a} d x^{\alpha}$ is a 1 -form in the adjoint representation of the gauge group. We shall write all group traces in the fundamental representation, and take the theory to be defined on $\mathbf{R}^{3}$, where the momentum space calculational procedure being used is valid. Our conventions are that the structure constants are real and completely antisymmetric, with $\left[T^{a}, T^{b}\right]=f^{a b c} T^{c}$. For the fundamental representation of $S U(n)$, the 

matrices $T^{a}$ are skew-hermitian and we take $\operatorname{tr} T^{a} T^{b}=-\frac{1}{2} \delta^{a b}$, while for the quadratic
Casimir we have $f^{a c d} f^{b c d}=c_{v} \delta^{a b}$.

The local symmetries of (1) correspond to the usual Yang-Mills gauge invariance and, in addition, a further local $\theta$-symmetry due to the presence of the $B$ field. Specifically,
we have

$$
\delta A_{\alpha}=D_{\alpha} \omega, \delta B_{\alpha}=D_{\alpha} \theta+\left[B_{\alpha}, \omega\right] .
$$

The first step in a one-loop background field calculation is to split the fields into a background plus a quantum part as follows:

$$
A \rightarrow A+A^{q}, B \rightarrow B+B^{q} .
$$

We stress that the backgrounds of interest here are necessarily off-shell, in compliance with the rules of the Kallosh theorem [6]. With this decomposition, the symmetries now read:

$$
\begin{aligned}
& \delta A_{\alpha}=D_{\alpha} \omega, \delta A_{\alpha}^{q}=\left[A_{\alpha}^{q}, \omega\right], \\
& \delta B_{\alpha}=D_{\alpha} \theta+\left[B_{\alpha}, \omega\right], \delta B_{\alpha}^{q}=\left[B_{\alpha}^{q}, \omega\right]+\left[A_{\alpha}^{q}, \theta\right],
\end{aligned}
$$

where the covariant derivative is with respect to the background field. In order to quantize the theory, we need a suitable set of gauge fixing conditions, where suitable in this instance is defined to mean that they are covariant with respect to (4). The following set can be deemed appropriate:

$$
G_{\phi} \equiv D \cdot A^{q}=0, G_{\pi} \equiv D \cdot B^{q}+\left[B_{\alpha}, A^{q \alpha}\right]=0,
$$

and indeed it can be checked that they transform as

$$
\delta G_{\phi}=\left[G_{\phi}, \omega\right], \delta G_{\pi}=\left[G_{\pi}, \omega\right]+\left[G_{\phi}, \theta\right] .
$$

Our aim now is to implement these covariant gauge fixing conditions in the quantum action with the use of two arbitrary gauge fixing parameters, which we denote by $\alpha$ and $\alpha^{\prime}$. The part of the quantum action which is quadratic in the quantum fields is given by

$$
\begin{aligned}
S_{q}^{(2)} & =\int d^{3} x \operatorname{tr}\left\{\epsilon^{\alpha \beta \gamma}\left(2 B_{\alpha}^{q} D_{\beta} A_{\gamma}^{q}+B_{\alpha}\left[A_{\beta}^{q}, A_{\gamma}^{q}\right]+\frac{k}{4 \pi} A_{\alpha}^{q} D_{\beta} A_{\gamma}^{a}\right)\right. \\
& \left.+2 \phi\left(D \cdot A^{q}-\frac{\alpha}{2} \phi\right)+2 \pi\left(D \cdot B^{q}+\left[B_{\alpha}, A^{q \alpha}\right]-\frac{\alpha^{\prime}}{2} \pi\right)\right\}+ \text { ghosts }
\end{aligned}
$$

where one recalls that the ghost and multiplier fields do not possess classical backgrounds. The "no VD" gauge corresponds to choosing both of these parameters to be zero. We are concerned here with the first order matrix $H_{a b}^{\alpha \beta}$ connecting the gauge fields and multipliers; specifically this is given by

$$
\begin{aligned}
S_{q}^{(2)} & =\frac{1}{2} \int d^{3} x\left(\begin{array}{llll}
B_{\alpha}^{q} & A_{\alpha}^{q} & \phi & \pi
\end{array}\right)^{a} \\
& \times\left(\begin{array}{cccc}
0 & -\epsilon^{\alpha \gamma \beta} D_{\gamma}^{a b} & 0 & D_{\alpha}^{a b} \\
-\epsilon^{\alpha \gamma \beta} D_{\gamma}^{a b} & -\epsilon^{\alpha \gamma \beta}\left(f^{a c b} B_{\gamma}^{c}+\frac{k}{4 \pi} D_{\gamma}^{a b}\right) & D_{\alpha}^{a b} & f^{a c b} B_{\alpha}^{c} \\
0 & -D_{\beta}^{a b} & \alpha \delta^{a b} & 0 \\
-D_{\beta}^{a b} & -f^{a c b} B_{\beta}^{c} & 0 & \alpha^{\prime} \delta^{a b}
\end{array}\right)\left(\begin{array}{c}
B_{\beta}^{q} \\
A_{\beta}^{q} \\
\phi \\
\pi
\end{array}\right)^{b} .
\end{aligned}
$$


Eqn. (8) defines a rank-2 symmetric object $H_{i j}$, which lies between the fields $\Phi^{i}$ and $\Phi^{j}$, where we adopt the collective notation $\Phi^{i} \equiv\left(B_{\alpha}^{q a}(x), A_{\alpha}^{q a}(x), \phi^{a}(x), \pi^{a}(x)\right)$.

The theory is defined via the partition function

$$
Z=\int \sqrt{\operatorname{det} G_{i j}} \prod_{i} d \Phi^{i} e^{i \Phi^{i} H_{i j} \Phi^{j}}
$$

the result of which is

$$
\operatorname{det}^{1 / 2}\left[G_{i j}\right] \operatorname{det}^{-1 / 2}\left[H_{i j}\right]=\operatorname{det}^{-1 / 2}\left[H_{j}^{i}\right]
$$

As shown in [3], the presence of the $\sqrt{\operatorname{det} G_{i j}}$ is crucial for ensuring the field reparametrization invariance of the path integral, or in other words, the background gauge invariance, even when this factor is field independent.

At this juncture, therefore, we must address the issue of which field space metric is appropriate. For the case of the gauge fields, we first re-write the transformations (2) in condensed notation $[4,5]$ as:

$$
\delta \phi^{i}=K_{\alpha}^{i} \epsilon^{\alpha} .
$$

Here, the field $\phi^{i}$ labels the classical fields $A$ and $B$, the symmetry generators are denoted by $K_{\alpha}^{i}$, and the infinitesimal gauge parameters are $\epsilon^{\alpha}=(\omega, \theta)$.

An acceptable field metric is defined by the requirement that it admits the gauge generators as Killing vectors $[4,5]$, that is

$$
0=G_{i k} \partial_{j} K_{\alpha}^{k}+G_{j k} \partial_{i} K_{\alpha}^{k}+\left(\partial_{k} G_{i j}\right) K_{\alpha}^{k},
$$

for all $\alpha$. In the case at hand, one can establish that a constant, field independent, metric which satisfies (12) does exist. However, we must also obtain a suitable metric on the multiplier space. Eqn. (6) defines the transformations of the gauge fixing conditions; together with the requirement that $\phi G_{\phi}+\pi G_{\pi}$ be invariant with respect to (4), we find that the multiplier fields transform as:

$$
\delta \phi=[\phi, \omega]+[\pi, \theta], \delta \pi=[\pi, \omega] .
$$

Given these transformations, we can determine the multiplier metric by requiring that $\delta\left(G_{\alpha \beta} \lambda^{\alpha} \lambda^{\beta}\right)=0$, where $\lambda^{\alpha} \equiv(\phi, \pi)$.

Under these conditions, the most general solution is given by

$$
G_{i j}=\left(\begin{array}{cccc}
0 & \lambda \delta_{\alpha \beta} & 0 & 0 \\
\lambda \delta_{\alpha \beta} & \sigma \delta_{\alpha \beta} & 0 & 0 \\
0 & 0 & 0 & \lambda^{\prime} \\
0 & 0 & \lambda^{\prime} & \sigma^{\prime}
\end{array}\right) \delta^{a b} \delta(x-y)
$$

where $\lambda$ and $\lambda^{\prime}$ are strictly non-zero. Re-scaling $A^{q}$ and $B^{q}$ by $1 / \sqrt{\lambda}$, and $\pi$ and $\phi$ by $1 / \sqrt{\lambda^{\prime}}$, we find that the inverse metric takes the form:

$$
G^{i j}=\left(\begin{array}{cccc}
-r \delta_{\alpha \beta} & \delta_{\alpha \beta} & 0 & 0 \\
\delta_{\alpha \beta} & 0 & 0 & 0 \\
0 & 0 & -r^{\prime} & 1 \\
0 & 0 & 1 & 0
\end{array}\right) \delta^{a b} \delta(x-y)
$$


with $r=\frac{\sigma}{\lambda}$ and $r^{\prime}=\frac{\sigma^{\prime}}{\lambda^{\prime}}$.

Having obtained all the necessary ingredients, we can now proceed with the calculation. In order to regularize (10) we define the $\zeta$ and $\eta$ functions of the operator $H \equiv H_{j}^{i}=$
$G^{i k} H_{k j}$ via its eigenvalues $\lambda_{n}$ :

$$
\zeta_{H}(s)=\sum_{n}\left|\lambda_{n}\right|^{-s}, \eta_{H}(s)=\sum_{n}\left(\operatorname{sign} \lambda_{n}\right)\left|\lambda_{n}\right|^{-s} .
$$

This leads to the result that

$$
Z_{r e g}=e^{\frac{1}{2} \zeta_{H}^{\prime}(0)+\frac{i \pi}{4} \eta_{H}(0)}
$$

We are interested in establishing a Kallosh theorem for the phase contribution to the path integral; this phase is given by the $\eta$-function which has the following integral
representation:

$$
\eta_{H}(s)=\frac{1}{\Gamma\left(\frac{s+1}{2}\right)} \int_{0}^{\infty} d t t^{(s-1) / 2} \operatorname{Tr}\left[H e^{-H^{2} t}\right] .
$$

In general, $\eta(s)$ is difficult to calculate for arbitrary values of $s$; fortunately, our interest is in its value at $s=0$ which has a more manageable representation. To see this, one decomposes $H$ as $H_{0}+H_{1}$, where $H_{0}$ is independent of the background fields, and $H_{1}$ contains the interaction terms. One can then employ a trick due to Gilkey [12], see for example [13], to show that

$$
\eta_{H}(0)=\lim _{s \rightarrow 0} \frac{-s}{\Gamma\left(\frac{s+1}{2}\right)} \int_{0}^{\infty} d t t^{(s-1) / 2}\left[T r_{0}+T r_{1}+T r_{2}+\cdots\right]
$$

where

$$
\begin{aligned}
& \operatorname{Tr}_{0}=\operatorname{Tr}\left[H_{1} e^{-H_{0}^{2} t}\right] \\
& \operatorname{Tr}_{1}=-t \int_{0}^{1} d u \operatorname{Tr}\left[e^{-H_{0}^{2} u t} H_{1} e^{-H_{0}^{2}(1-u) t}\left(\frac{1}{2}\left\{H_{0}, H_{1}\right\}+\frac{1}{3} H_{1}^{3}\right)\right], \\
& \operatorname{Tr}_{2}=\frac{t^{2}}{3} \int_{0}^{1} u d u \int_{0}^{1} d v \operatorname{Tr}\left[e^{-H_{0}^{2} u v t} H_{1} e^{-H_{0}^{2}(1-u) t}\left\{H_{0}, H_{1}\right\} e^{-H_{0}^{2} u(1-v) t}\left\{H_{0}, H_{1}\right\}\right] .
\end{aligned}
$$

The calculation thus amounts to an evaluation of the those terms which contribute a pole in s, so as to cancel the explicit $s$ factor in (19); these can be performed most readily in momentum space with the definition

$$
\operatorname{Tr}[\mathcal{O}]=\int \frac{d^{n} p}{(2 \pi)^{n}}\left\langle p\left|\operatorname{Tr}^{\prime} \mathcal{O}\right| p\right\rangle=\int \frac{d^{n} p}{(2 \pi)^{n}} d^{n} x d^{n} y\langle p \mid x\rangle\left\langle x\left|\operatorname{Tr}^{\prime} \mathcal{O}\right| y\right\rangle\langle y \mid p\rangle
$$

Here, the prime indicates a trace over any other indices carried by the operator $\mathcal{O}$. Our conventions are such that $\langle p \mid x\rangle=e^{-i p x}$ and $A(p)=\int d^{n} x e^{-i p x} A(x)$.

\section{A Kallosh Theorem}

From (8) and (15), we see that we must now compute the $\eta$-function for the operator 


$$
\begin{aligned}
& \left(G^{i k} H_{k j}\right)_{\alpha \beta}^{a b}(x, y) \\
& =\left(\begin{array}{cccc}
-\epsilon^{\alpha \gamma \beta} D_{\gamma x}^{a b} & \epsilon^{\alpha \gamma \beta}\left(\hat{r} D_{\gamma x}^{a b}-f^{a c b} B_{\gamma x}^{c}\right) & D_{\alpha x}^{a b} & -r D_{\alpha x}^{a b}+f^{a c b} B_{\alpha x}^{c} \\
0 & -\epsilon^{\alpha \gamma \beta} D_{\gamma x}^{a b} & 0 & D_{\alpha x}^{a b} \\
-D_{\beta x}^{a b} & r^{\prime} D_{\beta x}^{a b}-f^{a c b} B_{\beta x}^{c} & -r^{\prime} \alpha \delta^{a b} & \alpha^{\prime} \delta^{a b} \\
0 & -D_{\beta x}^{a b} & \alpha \delta^{a b} & 0
\end{array}\right) \delta(x-y),
\end{aligned}
$$

where $\hat{r}=r-\frac{k}{4 \pi}$. In momentum space, we have the following representations for the free $\left(H_{0}\right)$ and interacting $\left(H_{1}\right)$ parts of $H$ :

$$
\left\langle p\left|H_{0}\right| q\right\rangle_{a b}^{\alpha \beta}=i \delta_{a b}(p-q)\left(\begin{array}{cccc}
-\epsilon^{\alpha \gamma \beta} p_{\gamma} & \hat{r} \epsilon^{\alpha \gamma \beta} p_{\gamma} & p_{\alpha} & -r p_{\alpha} \\
0 & -\epsilon^{\alpha \gamma \beta} p_{\gamma} & 0 & p_{\alpha} \\
-p_{\beta} & r^{\prime} p_{\beta} & i r^{\prime} \alpha & -i \alpha^{\prime} \\
0 & -p_{\beta} & -i \alpha & 0
\end{array}\right)
$$

and

$$
\left\langle p\left|H_{1}\right| q\right\rangle_{a b}^{\alpha \beta}=f^{a c b}\left(\begin{array}{cccc}
-\epsilon^{\alpha \gamma \beta} A_{\gamma}^{c} & \epsilon^{\alpha \gamma \beta}\left(\hat{r} A_{\gamma}^{c}-B_{\gamma}^{c}\right) & A_{\alpha}^{c} & \left(-r A_{\alpha}^{c}+B_{\alpha}^{c}\right) \\
0 & -\epsilon^{\alpha \gamma \beta} A_{\gamma}^{c} & 0 & A_{\alpha}^{c} \\
-A_{\beta}^{c} & \left(r^{\prime} A_{\beta}^{c}-B_{\beta}^{c}\right) & 0 & 0 \\
0 & -A_{\beta}^{c} & 0 & 0
\end{array}\right),
$$

where $A$ above implicitly denotes $A(p-q)$. The next step is to determine the structure of $H_{0}^{2}$, and one finds

$$
\left\langle p\left|H_{0}^{2}\right| q\right\rangle_{a b}^{\alpha \beta}=\left(p^{2} I^{\alpha \beta}+X^{\alpha \beta}(p)\right) \delta_{a b} \delta(p-q),
$$

where

$$
X^{\alpha \beta}(p)=\left(\begin{array}{cccc}
0 & {\left[-2 \hat{r}\left(\delta_{\alpha \beta} p^{2}-p_{\alpha} p_{\beta}\right)\right.} & -i \alpha\left(r+r^{\prime}\right) p_{\alpha} & i \alpha^{\prime} p_{\alpha} \\
0 & \left.-\left(r+r^{\prime}\right) p_{\alpha} p_{\beta}\right] & & \\
0 & 0 & i \alpha p_{\alpha} & 0 \\
i \alpha r^{\prime} p_{\beta} & -i \alpha^{\prime} p_{\beta}-i \alpha r^{\prime 2} p_{\beta} & \alpha^{2} r^{\prime 2}+\alpha \alpha^{\prime} & -\left(r+r^{\prime}\right) p^{2}-r^{\prime} \alpha \alpha^{\prime} \\
-i \alpha p_{\beta} & i \alpha r^{\prime} p_{\beta} & -\alpha^{2} r^{\prime} & \alpha \alpha^{\prime}
\end{array}\right)
$$

The important point to notice here is that $H_{0}^{2}$ is not diagonal; some care is then needed in order to deal with the factors of $e^{-H_{0}^{2} t}$ which appear in the traces in (20).

For the purposes of verifying the Kallosh theorem, it suffices to consider the $H_{1}\left\{H_{0}, H_{1}\right\}$ part of $T r_{1}$ in (20). This can be seen by using a simple power counting argument, where one observes that the operator $\partial$ produces terms of order $t^{-1 / 2}$. Equivalently, each power of momentum $p$ is of order $t^{-1 / 2}$. In performing the relevant trace, one encounters the exponential factor $e^{-H_{0}^{2} t}$ in various combinations. An understanding of the structure of this term is therefore important for determining which terms have the potential of contributing a pole in the trace. Given (25), one can expand the exponential in powers of $X$. However, using the power counting argument one can see that it is only necessary to retain terms in this expansion which are at most $O\left(t^{1 / 2}\right)$; higher order terms do not contribute a pole in $s$. Due to the presence of $O\left(t^{0}\right)$ terms in $(26)$, it is necessary to 
expand the exponential to third order. Fortunately, the matrix structure of $X$ contrives to terminate the series at this point. The relevant piece is then given by

where

$$
\left\langle p\left|e^{-H_{0}^{2} t}\right| q\right\rangle_{a b}^{\alpha \beta}=e^{-p^{2} t}\left(I^{\alpha \beta}+\bar{X}^{\alpha \beta}(p)\right) \delta_{a b} \delta(p-q),
$$

$$
\bar{X}^{\alpha \beta}(p)=\left(-X t+X^{2} \frac{t^{2}}{2 !}-X^{3} \frac{t^{3}}{3 !}\right)^{\alpha \beta}(p) .
$$

The non-zero entries in $\bar{X}$ are as follows:

$$
\begin{array}{rll}
(1,2) & : & 2 \hat{r} t\left(\delta_{\alpha \beta} p^{2}-p_{\alpha} p_{\beta}\right)+\left(r+r^{\prime}\right) t p_{\alpha} p_{\beta} \\
(1,3): & i \alpha t\left(r+r^{\prime}\right) p_{\alpha}\left[1-\frac{t}{2} p^{2}\right] \\
(1,4): & -i \alpha^{\prime} t p_{\alpha}+i \alpha \frac{t^{2}}{2} p^{2} p_{\alpha}\left(r+r^{\prime}\right)^{2}\left(1-\frac{t}{3} p^{2}\right) \\
(2,3): & -i \alpha t p_{\alpha} \\
(2,4): & -i \alpha \frac{t^{2}}{2} p^{2} p_{\alpha}\left(r+r^{\prime}\right) \\
(3,1): & -i \alpha t p_{\beta}\left[r^{\prime}-\frac{t}{2}\left(r+r^{\prime}\right) p^{2}\right] \\
(3,2): & i \alpha^{\prime} t p_{\beta}+i \alpha t p_{\beta}\left[r^{2}-t p^{2} r^{\prime}\left(r+r^{\prime}\right)+\frac{t^{2}}{6} p^{4}\left(r+r^{\prime}\right)^{2}\right] \\
(3,4): & \left(r+r^{\prime}\right) t p^{2} \\
(4,1): & i \alpha t p_{\beta} \\
(4,2): & -i \alpha t p_{\beta}\left[r^{\prime}-\frac{t}{2} p^{2}\left(r+r^{\prime}\right)\right] .
\end{array}
$$

It remains only to compute the trace. Upon expanding the exponential factors, one finds that there are four pieces which contribute non-zero pole terms. These can be seen upon examination of the relevant trace, namely

$$
\begin{aligned}
\operatorname{Tr}_{1} & =-\frac{t}{2} \int_{0}^{1} d u d^{3} p d^{3} k e^{-p^{2} u t-(p-k)^{2}(1-u) t} \\
& \cdot\left\langle p\left|(I+\bar{X}) H_{1}(I+\bar{X})\right| p-k\right\rangle_{a b}^{\alpha \beta}\left\langle p-k\left|\left\{H_{0}, H_{1}\right\}\right| p\right\rangle_{b a}^{\beta \alpha} .
\end{aligned}
$$

The contributions to $T r_{1}$ are listed in turn as:

$$
\begin{aligned}
H_{1} & \rightarrow\left[-A^{a}(k) \cdot A^{a}(-k)\left(2 r+4 r^{\prime}\right)+4 A^{a}(k) \cdot B^{a}(-k)\right], \\
\bar{X} H_{1} & \rightarrow\left[A^{a}(k) \cdot A^{a}(-k)\left(7 u r+9 u r^{\prime}-5 u^{2}\left(r+r^{\prime}\right)\right)-4 u A^{a}(k) \cdot B^{a}(-k)\right], \\
H_{1} \bar{X} & \rightarrow\left[A^{a}(k) \cdot A^{a}(-k)\left(2 r+4 r^{\prime}+3 u r+u r^{\prime}-5 u^{2}\left(r+r^{\prime}\right)\right)\right. \\
& \left.+4(u-1) A^{a}(k) \cdot B^{a}(-k)\right], \\
\bar{X} H_{1} \bar{X} & \rightarrow\left[A^{a}(k) \cdot A^{a}(-k)\left(-10 u\left(r+r^{\prime}\right)+10 u^{2}\left(r+r^{\prime}\right)\right)\right],
\end{aligned}
$$

where each piece in (31) is multiplied by

$$
-\frac{\alpha c_{V} t^{-1 / 2}}{2(4 \pi)^{3 / 2}} \int_{0}^{1} d u d^{3} k e^{-k^{2} u(1-u) t}
$$


and $\bar{X}$ on the left, and right, in (31) correspond to (28) with the replacements $t \rightarrow u t$, and $t \rightarrow(1-u) t$, respectively.

We can now sum the different contributions, and one finds that all the $\alpha$ terms which are present do indeed sum to zero. Perhaps it is worth remarking the absence of any $\alpha^{\prime}$ and $B \cdot B$ terms in the above calculation. In addition, the result is independent of the scale parameters $r, r^{\prime}$, and $\hat{r}$. We have thus verified the Kallosh theorem for the $\eta$-function phase of this theory. In other words, the $\eta$-function contribution to the off-shell effective action is independent of the gauge fixing parameters $\alpha$ and $\alpha^{\prime}$. The result thus coincides with the 'no VD' gauge result obtained in $[1,3]$, namely

$$
\eta_{H}(s=0)=\frac{2 c_{v}}{\pi^{2}} \int d^{3} x \operatorname{tr} \epsilon^{\alpha \beta \gamma}\left(A_{\alpha} \partial_{\beta} A_{\gamma}+\frac{2}{3} A_{\alpha} A_{\beta} A_{\gamma}\right)
$$

\section{Generalized BF Theory}

One can also consider a generalized system with classical action [11]

$$
\begin{aligned}
S & =\frac{1}{2 \pi} \int d^{3} x \epsilon^{\alpha \beta \gamma} \operatorname{tr}\left[\frac{1}{2} B_{\alpha} F_{\beta \gamma}+\frac{1}{3} B_{\alpha} B_{\beta} B_{\gamma}\right] . \\
& +\frac{1}{4 \pi} \int d^{3} x \epsilon^{\alpha \beta \gamma} \operatorname{tr}\left[A_{\alpha} \partial_{\beta} A_{\gamma}+\frac{2}{3} A_{\alpha} A_{\beta} A_{\gamma}+B_{\alpha} D_{\beta} B_{\gamma}\right] \equiv S_{1}+S_{2} .
\end{aligned}
$$

In this case, the local symmetries are given by

$$
\delta A_{\alpha}=D_{\alpha} \omega+\left[B_{\alpha}, \theta\right], \delta B_{\alpha}=D_{\alpha} \theta+\left[B_{\alpha}, \omega\right],
$$

with the following set of covariant gauge fixing conditions:

$$
G_{\phi} \equiv D \cdot A^{q}+\left[B_{\alpha}, B^{q \alpha}\right], G_{\pi} \equiv D \cdot B^{q}+\left[B_{\alpha}, A^{q \alpha}\right],
$$

These transform as:

$$
\delta G_{\phi}=\left[G_{\phi}, \omega\right]+\left[G_{\pi}, \theta\right], \delta G_{\pi}=\left[G_{\pi}, \omega\right]+\left[G_{\phi}, \theta\right] .
$$

Following the same strategy as was employed in the previous example, we find the transformations of the multiplier fields:

$$
\delta \phi=[\phi, \omega]+[\pi, \theta], \delta \pi=[\pi, \omega]+[\phi, \theta] .
$$

The resulting field space metric turns out to be of the general form

$$
G_{i j}=\left(\begin{array}{cccc}
\sigma & \lambda & 0 & 0 \\
\lambda & \sigma & 0 & 0 \\
0 & 0 & \sigma^{\prime} & \lambda^{\prime} \\
0 & 0 & \lambda^{\prime} & \sigma^{\prime}
\end{array}\right)
$$

where the only restriction is that $\sigma^{2}-\lambda^{2} \neq 0$, and similarly for the primed variables. Various studies of the effective action for this model have been performed in [1] and [15]. In particular, the system $S_{1}$ was studied in [15] with a diagonal field space metric, $\sigma=\sigma^{\prime}=1$ and $\lambda=\lambda^{\prime}=0$. It is clear that such a metric is an acceptable Killing 
metric for this theory. However, the above analysis indicates that there is more freedom here in choosing a field metric, and different choices will lead to differing results for the eta-function of the theory. For example, one may study the $S_{1}$ system with $\sigma=\sigma^{\prime}=0$, and $\lambda=\lambda^{\prime}=1$. One can then check that the phase contribution is proportional to the $S_{2}$ action. Such a situation simply means that the quantum theory under study depends on the choice of field metric, and different choices can lead to inequivalent quantum theories; see [11] in this regard.

\section{Acknowledgements}

S.M. appreciates the hospitality of the Theory Division at CERN.

\section{References}

[1] I. Oda and S. Yahikozawa, Effective actions of $2+1$ dimensional gravity and BF theory, ICTP preprint, IC/90/44, April 1990.

[2] D. Birmingham, H.T. Cho, R. Kantowski and M. Rakowski, Phys. Lett. 264B (1991) 324.

[3] D. Birmingham, H.T. Cho, R. Kantowski and M. Rakowski, Operator phases in BFtype topological field theories II, CERN preprint, CERN-TH. 6160/91, July 1991, Phys. Lett. B, to appear.

[4] G. Vilkovisky, Nucl. Phys. B234 (1984) 125.

[5] B. DeWitt, in Architecture of Fundamental Interactions at Short Distances, proceedings of Les Houches Summer School 1985, edited by P. Ramond and R. Stora, North-Holland (1987).

[6] R. Kallosh, Nucl. Phys. B78 (1974) 293.

[7] G.T. Horowitz, Commun. Math. Phys. 125 (1989) 417.

[8] M. Blau and G. Thompson, Ann. Phys. 205 (1991) 130.

[9] A. Karlhede and M. Roček, Phys. Lett. 224B (1989) 58; R.C. Myers and V. Periwal, Phys. Lett. 225B (1989) 352.

[10] E. Witten, Commun. Math. Phys. 121 (1989) 351.

[11] E. Witten, Nucl. Phys. B311 (1988) 46.

[12] P.B. Gilkey, Invariance Theory, the Heat Equation and the Atiyah-Singer Index Theorem, Publish or Perish Inc. (1984). [13] D. Birmingham, H.T. Cho, R. Kantowski and M. Rakowski, Phys. Rev. D42 (1990)
3476. [14] S.R. Huggins, G. Kunstatter, H.P. Leivo and D.J. Toms, Nucl. Phys. B301 (1987)
627 .

[15] D. Birmingham, R. Gibbs and S. Mokhtari, Phys. Lett. 263B (1991) 176. 